\title{
Inhibition of myostatin protects against diet-induced obesity by enhancing fatty acid oxidation and promoting a brown adipose phenotype in mice
}

\author{
C. Zhang $\cdot$ C. McFarlane $\cdot$ S. Lokireddy $\cdot$ S. Masuda $\cdot$ \\ X. Ge • P. D. Gluckman • M. Sharma • R. Kambadur
}

Received: 4 July 2011 / Accepted: 29 July 2011 /Published online: 17 September 2011

(C) Springer-Verlag 2011

\begin{abstract}
Aims/hypothesis Although myostatin-null $\left(\mathrm{Mstn}^{-/-}\right)$mice fail to accumulate fat in adipose tissue when fed a highfat diet (HFD), little is known about the molecular mechanism(s) behind this phenomenon. We therefore sought to identify the signalling pathways through which myostatin regulates accumulation and/or utilisation of fat. Methods Wild-type, $\mathrm{Mstn}^{-1-}$ and wild-type mice treated with soluble activin type IIB receptor (sActRIIB) were fed a control chow diet or an HFD for 12 weeks. Changes in gene expression were measured by microarray and quantitative PCR. Histological changes in white adipose tissue were assessed together with peripheral tissue fatty acid oxidation and changes in circulating hormones following HFD feeding. Results Our results demonstrate that inactivation of myostatin results in reduced fat accumulation in mice
\end{abstract}

C. Zhang and C. McFarlane contributed equally to this manuscript.

Electronic supplementary material The online version of this article (doi:10.1007/s00125-011-2304-4) contains peer-reviewed but unedited supplementary material, which is available to authorised users.

C. Zhang · C. McFarlane · S. Masuda • P. D. Gluckman •

R. Kambadur

Singapore Institute for Clinical Sciences, Agency for Science,

Technology and Research (A*STAR),

Brenner Centre for Molecular Medicine,

Singapore, Republic of Singapore

C. Zhang $\cdot$ S. Lokireddy $\cdot$ X. Ge $\cdot$ R. Kambadur $(\bowtie)$

School of Biological Sciences, 60 Nanyang Drive,

Nanyang Technological University,

Singapore 637551, Republic of Singapore

e-mail: KRavi@ntu.edu.sg

\section{Sharma}

Department of Biochemistry, YLL School of Medicine,

National University of Singapore,

Singapore, Republic of Singapore on an HFD. Molecular analysis revealed that metabolic benefits, due to lack of myostatin, are mediated through at least two independent mechanisms. First, lack of myostatin increased fatty acid oxidation in peripheral tissues through induction of enzymes involved in lipolysis and in fatty acid oxidation in mitochondria. Second, inactivation of myostatin also enhanced brown adipose formation in white adipose tissue of $\mathrm{Mstn}^{-/}$ mice. Consistent with the above, treatment of HFD-fed wild-type mice with the myostatin antagonist, sActRIIB, reduced the obesity phenotype.

Conclusions/interpretation We conclude that absence of myostatin results in enhanced peripheral tissue fatty acid oxidation and increased thermogenesis, culminating in increased fat utilisation and reduced adipose tissue mass. Taken together, our data suggest that anti-myostatin therapeutics could be beneficial in alleviating obesity.

Keywords Brown adipose tissue $\cdot$ COX-2 . Fatty acid oxidation · Myostatin · PPAR $\beta$ - Prostaglandin · sActRIIB . UCP1 1 White adipose tissue

$\begin{array}{ll}\text { Abbreviations } \\ \text { AMPK } & \text { AMP-activated protein kinase } \\ \text { BAT } & \text { Brown adipose tissue } \\ \text { COX-2 } & \text { Cyclooxygenase-2 } \\ \text { HFD } & \text { High-fat diet } \\ \text { Mstn }^{--} & \text {Myostatin-null } \\ \text { PGE2 } & \text { Prostaglandin } E_{2} \\ \text { PGF1 } \alpha & \text { 6-Keto-prostaglandin } \text { F }_{1 \alpha} \\ \text { PPAR } & \text { Peroxisome proliferator-activated receptor } \\ \text { qPCR } & \text { Quantitative real-time PCR } \\ \text { sActRIIB } & \text { Soluble activin type IIB receptor } \\ \text { UCP } & \text { Uncoupling protein } \\ \text { WAT } & \text { White adipose tissue }\end{array}$




\section{Introduction}

Myostatin, a secreted growth factor and member of the TGF- $\beta$ superfamily, has recently emerged as an important growth factor that not only regulates levels of lean muscle mass, but also body fat content in mice. While high levels of myostatin are detected in skeletal muscle [1], low levels are also detected in other tissues, including adipose tissue [1] and heart [2]. Inactivation of the Mstn gene [1] in mice, or mutations in the bovine [3, 4], ovine [5] and human MSTN [6] genes result in a similar phenotype of increased muscle growth. In addition murine studies have reported significant decreases in the amount of adipose tissue in association with loss of myostatin function. For example, McPherron and Lee reported that myostatin-null $\left(\mathrm{Msth}^{-/-}\right)$ mice, despite having normal food intake and body temperature, and a reduced metabolic rate, have significantly less mean total body fat and lower serum leptin levels than wild-type mice [7]. Furthermore, loss of myostatin in genetic models of obesity (agouti lethal yellow and leptindeficient mice $\left[L e p^{o b / o b}\right]$ ) suppressed body fat accumulation [7]. Similarly, myostatin prodomain-overexpressing transgenic mice (in which myostatin function is blocked) also displayed significantly decreased fat-pad weight and were also resistant to obesity induced by a high-fat diet (HFD) [8]. These results suggest that loss of functional myostatin, while increasing muscle mass, decreases body fat accumulation. In contrast, Feldman et al. reported that ectopic production of myostatin, specifically in adipose tissue, induces adipose wasting [9]. Therefore the exact role of myostatin in regulating adipose tissue growth and functionality remains to be fully understood.

Increased peripheral tissue fatty acid oxidation can lead to increased energy expenditure to protect against obesity. Adipose tissue plays a critical function in this regard, since white adipose tissue (WAT) acts as an energy-storing organ and brown adipose tissue (BAT) acts as an energyconsuming organ. White adipocytes are characterised by a large unilocular lipid droplet, whereas brown adipocytes contain several relatively small lipid droplets, giving them a characteristic multilocular appearance. Brown adipocytes also contain large numbers of mitochondria with densely packed, regularly arranged cristae [10], a characteristic of high mitochondrial activity. BAT is an important regulator of thermogenesis, in fact BAT has a high abundance of uncoupling protein (UCP) 1, which functions to uncouple oxidative phosphorylation, resulting in release of excess energy as heat to maintain normal body temperature. Furthermore, the expression of genes including Dio2, Cidea and Pgc-1 $\alpha$ (also known as Ppargcla), as well as that of genes encoding members of the peroxisome proliferatoractivated receptor (PPAR) family, is also highly enriched in BAT. Interestingly, differentiated WAT has been shown to acquire BAT-like properties; in particular, exposure to cold or stimulation with a $\beta 3$-adrenergic agonist can induce the emergence of multilocular, UCP1-producing, brown adipocyte depots in WAT [11-13].

Here, we have identified molecular mechanisms that promote leanness in the absence of myostatin. We show that lack of myostatin promotes increased fatty acid oxidation in peripheral tissues, as well as increased prevalence of Brown-like adipose tissue in WAT. As a result, we show that $\mathrm{Mstn}^{-/-}$mice are resistant to obesity even when challenged with an HFD. We further show that treatment of mice with a myostatin antagonist, soluble activin type IIB receptor (sActRIIB), can protect mice from dietinduced obesity. These data suggest that novel therapeutics designed to increase body energy expenditure could be viable as anti-obesity drugs.

\section{Methods}

Animals Animal experiments were conducted with approval from the Institutional Animal Care and Use Committee, Singapore. Wild-type mice (C57BL/6 background) were supplied by the Centre for Animal Resources, National University of Singapore, Singapore. Msth $^{-/}$mice (C57BL/6 background) were donated by S.-J. Lee (Department of Molecular Biology \& Genetics, Johns Hopkins University School of Medicine, Baltimore, MD, USA). Mstn ${ }^{-1}$ and wild-type mice were housed in groups at a constant temperature $\left(20^{\circ} \mathrm{C}\right)$ under a $12 / 12 \mathrm{~h}$ artificial light/dark cycle with free access to water. Two groups of 7-week-old male mice, consisting of five wild-type and five $\mathrm{Mstn}^{-/-}$mice each, were either fed an HFD (45\% energy from fat; 58V8; TestDiet, Richmond, IN, USA) or chow diet (10\% energy from fat; 58Y2; TestDiet) for 12 weeks. For sActRIIB antagonist studies, 7-week-old male wild-type mice were fed a chow diet or an HFD as above for a period of 12 weeks, together with intraperitoneal injection of saline or sActRIIB ( $5 \mu \mathrm{g} / \mathrm{g}$ body weight) administered three times per week. The generation and purification of the sActRIIB has been previously described in detail [14]. Body weight and energy intake (energy intake $=$ food consumption $\times$ metabolisable energy content) were recorded twice a week in all mice. Rectal temperatures of wild-type and $\mathrm{Mstn}^{--}$mice were measured using a rectal thermometer (Thermocouple; Kent Scientific, Torrington, CT, USA). All measurements and analyses were performed on epididymal WAT (gonadal) and M. gastrocnemius skeletal muscle unless otherwise stated.

RNA extraction and quantitative real-time PCR RNA extraction and quantitative real-time PCR (qPCR) were performed as previously described [14]; see Electronic supplementary material (ESM) for details. 
Measurement of triacylglycerol in WAT Lipid was extracted from peripheral tissues using a modified version of the Folch extraction method, as previously described $[15,16]$ (see ESM for details).

Measurement of serum NEFA and leptin, and total blood triacylglycerol Total blood triacylglycerol levels were analysed using an Accutrend Plus meter (Roche Diagnostics, Indianapolis, IN, USA). Serum NEFA was measured using a kit (NEFA-C kit; Wako, Chuo-Ku, Osaka, Japan). Serum leptin levels were also determined using a kit (Mouse Leptin ELISA; Millipore, Billerica, MA, USA), as per the manufacturer's instructions.

Haematoxylin and eosin staining of WAT Epididymal fatpads were fixed for $48 \mathrm{~h}$ with $10 \%$ (vol./vol.) formalin, then dehydrated in an automated tissue processor (TP1020; Leica, Wetzlar, Germany). The fat-pads were paraffinembedded, sectioned using a rotary microtome (RM2265; Leica) and stained with haematoxylin and eosin as previously described [17].

Immunohistochemical staining Immunohistochemical staining of fat-pad sections was performed using a kit (Vectastain Elite ABC; Vector Laboratories, Burlingame, CA, USA) according to the manufacturer's instructions with some modifications (see ESM for details).

Measurement of prostaglandin released from WAT explants Assessment of the levels of prostaglandins released from WAT explants was performed as previously described [13] (see ESM for details).

Assessment of fatty acid oxidation in tissues and myotube cultures Fatty acid oxidation in liver, WAT [18] and myotube cultures [19] was analysed as previously described (see ESM for details).

Microarray analysis Microarray analysis was performed in duplicate from WAT tissue isolated from wild-type and $\mathrm{Mstn}^{-/-}$mice. Gene expression changes identified by microarray were subsequently verified by $\mathrm{qPCR}$ in six different samples per group. RNA from WAT was isolated using TRIzol (Invitrogen, Carlsbad, CA, USA) according to the manufacturer's guidelines. RNA integrity was verified using a kit (Agilent RNA 6000 Nano Kit and 2100 Bioanalyzer; Agilent, Santa Clara, CA, USA). Microarray analysis was performed by Genomax Technologies, Singapore, as per their standardised techniques, using a mouse gene expression array (SurePrint G3; Agilent). Normalised data were used for pairwise comparisons between wild-type and $\mathrm{Mstn}^{-/-}$mice fed on chow diet. The resulting gene list from the pairwise comparisons included the genes with a value of $p \leq 0.05$ when using a standard two-tailed unequal variance $t$ test. All statistical analysis on the microarray data was done using GenSpring software (version 11.0.2; Agilent). Significantly upregulated genes ( $\geq 1.5$-fold) were subsequently verified via $\mathrm{qPCR}$.

Statistical analysis Multiple comparisons were made using two-way ANOVA and robust regression analysis. Single comparisons were made using two-tailed Student's $t$ tests. Data are expressed as mean \pm SEM and values of $p<0.05$ were considered significant. Experimental replicates were as described in relevant figures.

\section{Results}

$\mathrm{Mstn}^{-1-}$ mice exhibit reduced adiposity even when fed an HFD Recently we [14] and others [17, 20] have shown that chronic HFD feeding of $\mathrm{Mstn}^{-/-}$mice resulted in significantly less accumulation of visceral adipose tissue mass and improved resistance to diet-induced obesity when compared with wild-type mice on an HFD. To study this in further detail, we fed wild-type and $\mathrm{Mstn}^{-/-}$mice an HFD or chow diet for 12 weeks. While no significant difference was observed in the energy intake between wild-type mice fed an HFD or a chow diet, $\mathrm{Mstn}^{-/-}$mice on an HFD had increased energy intake over and above that of their chow diet-fed counterparts (Fig. 1a). As expected, wild-type mice fed an HFD showed a greater increase $(\sim 40 \%)$ in body weight than wild-type mice on a chow diet $(\sim 23 \%)$ after 12 weeks (Fig. 1b). In contrast, no significant difference in body weight was observed between $\mathrm{Mstn}^{-1-}$ mice fed a chow diet or those fed an HFD, despite the increased energy intake observed in the latter (Fig. 1b). Subsequent quantification of adipocyte area revealed that adipocytes from $\mathrm{Msth}^{-/-}$mice WAT were smaller $\left(<350 \mu \mathrm{m}^{2}\right)$ than those from wild-type mice, where the majority ranged from 350 to $700 \mu \mathrm{m}^{2}$ or were greater than $700 \mu \mathrm{m}^{2}$ (Fig. 1d). Furthermore, HFD feeding caused significant hypertrophy of adipocytes in wild-type mice, while insignificant hypertrophy was observed in $\mathrm{Mstn}^{-/-}$adipocytes (Fig. 1d). Consistent with the reduced adipose content, we found significantly lower triacylglycerol accumulation in $\mathrm{Mstn}^{-/}$ WAT than in wild-type WAT (Fig. 1e). We also observed reduced serum leptin levels in $\mathrm{Mstn}^{-/-}$compared with wildtype mice (Fig. 1f). These data suggest that fat accumulation in WAT in $\mathrm{Mstn}^{-1-}$ mice is reduced. We hypothesised that this could result from reduced triacylglycerol synthesis or enhanced fatty acid oxidation.

Lack of myostatin increases lipolysis and peripheral tissue fatty acid $\beta$-oxidation Microarray analysis revealed elevated expression of genes encoding enzymes involved in fatty acid 

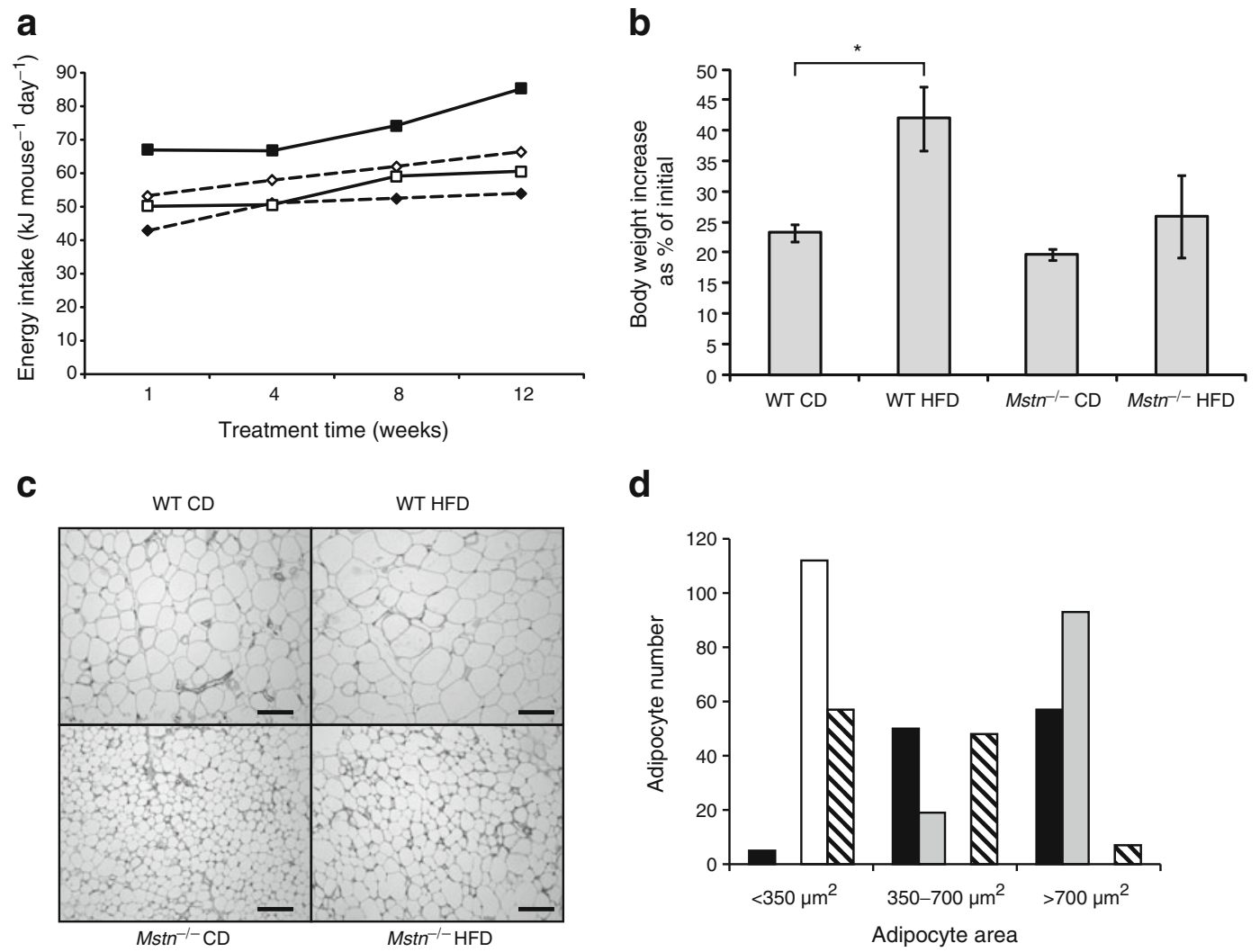

d
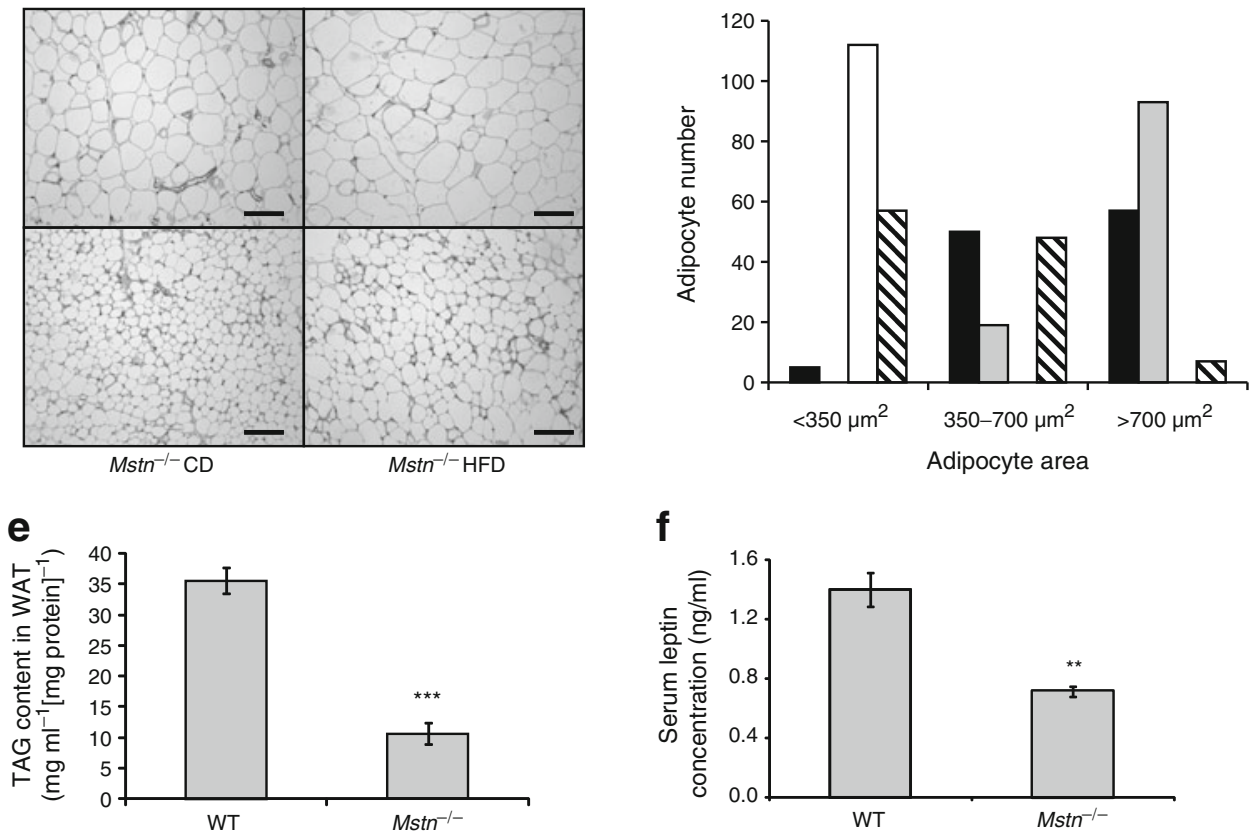

$\mathbf{f}$

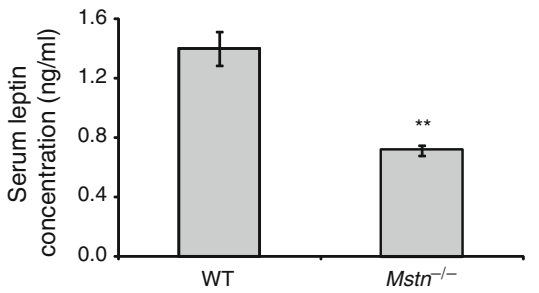

Fig. 1 Absence of myostatin results in a lean phenotype in mice. a Energy intake (in kilojoules) of wild-type and $\mathrm{Mstn}^{-/-}$mice during 12 weeks of chow diet and HFD treatment (black diamonds, wild-type chow diet; white squares, wild-type HFD; white diamonds, Mstn ${ }^{-1-}$ chow diet; black squares, $M s t n^{-/-}$HFD). b Body weight increase (as percentage of initial body weight) in wild-type (WT) and $\mathrm{Mstn}^{-/-}$mice after 12 weeks of chow diet (CD) and HFD treatment. c Haematoxylin and eosin staining (scale bars $100 \mu \mathrm{m}$, magnification $\times 10$ ) and $(\mathbf{d})$

synthesis, including Fasn and Acaca, in WAT tissue isolated from $\mathrm{Mstn}^{-/}$mice compared with wild-type mice WAT (Table 1). Subsequent qPCR analysis confirmed upregulation of Fasn and Acaca expression in $\mathrm{Mstn}^{-1-}$ WAT (Fig. 2a, b). Several-fold upregulation of genes that code for proteins involved in thermogenesis (Ucp1, Ucp2, Cidea, Cpt1b, Dio2), BAT differentiation (Cebpb) and prostaglandin synthesis (Ptgs2, Ptges and Ptges2) was also seen in $\mathrm{Msth}^{-/-}$WAT (Table 1). Furthermore, qPCR analysis revealed that genes encoding the lipolytic enzymes adipose triacylglycerol lipase, hormone sensitive lipase and monoacylglycerol lipase, which are critical for

quantification of adipocyte size from WAT of wild-type and $\mathrm{Mstn}^{-/-}$ mice after 12 weeks of chow diet and HFD treatment. Black bars, wild-type chow diet; grey bars, wild-type HFD; white bars, $\mathrm{Mstn}^{-/}$ chow diet; hatched bars, $\mathrm{Mstn}^{-/-}$HFD. e Total triacylglycerol (TAG) content in WAT (epididymal) isolated from wild-type and $\mathrm{Mstn}^{-1}$ mice, normalised to protein content. f Total serum leptin levels in wild-type and $\mathrm{Mstn}^{-/-}$mice. Values are mean \pm SEM (error bars); $n=5$; ${ }^{*} p<0.05, * * p<0.01, * * * p<0.001$

catalysing hydrolysis of triacylglycerol, diacylglycerol and monoacylglycerol respectively, were upregulated in WAT isolated from $\mathrm{Mstn}^{-/-}$mice (Fig. 2c-e). Importantly, the expression of genes involved in fatty acid oxidation (Ppara, Pparg, Ppargcla, Ppargclb, Acadvl, Acadm, Hadha, Acadl, Hadh, Hadhb, Acsl1 and Acsm3) was also enhanced in $\mathrm{Mstn}^{-/-}$WAT (Table 1). Moreover, qPCR analysis confirmed significant upregulation of $\mathrm{Acadl}$, Acsl6 and Acadvl in WAT (Fig. 3a-c), BAT (Fig. 3d-f) and skeletal muscle (ESM Fig. 1a-c) from chow diet-fed $\mathrm{Msth}^{-/-}$mice compared with wild-type mice on a chow diet. Importantly, while increased expression of Acadvl, 
Table 1 Global gene expression changes between WAT from wild-type and $\mathrm{Mstn}^{-/-}$mice fed on chow diet

\begin{tabular}{|c|c|c|c|}
\hline Genes by function & Gene name & Accession no. & Fold upregulation \\
\hline \multicolumn{4}{|l|}{ Fatty acid oxidation } \\
\hline Ppara/Ppar $\alpha$ & Peroxisome proliferator-activated receptor alpha & NM_011144 & 2.1 \\
\hline Pparg/Ppar $\gamma$ & Peroxisome proliferator-activated receptor gamma & NM_011146 & 2.0 \\
\hline Ppargcla/Pgc-1 $\alpha$ & Peroxisome proliferator-activated receptor, gamma, coactivator 1 alpha & NM_008904 & 2.2 \\
\hline Ppargclb & Peroxisome proliferator-activated receptor, gamma, coactivator 1 beta & NM_133249 & 4.0 \\
\hline Acadvl/Vlcad & Acyl-coenzyme A dehydrogenase, very long chain & NM_017366 & 1.7 \\
\hline Acadm & Acyl-coenzyme A dehydrogenase, medium chain & NM_007382 & 1.8 \\
\hline Hadha & Hydroxyacyl-coenzyme A dehydrogenase, alpha subunit & NM_178878 & 1.9 \\
\hline Acadl/Lcad & Acyl-coenzyme A dehydrogenase, long-chain & NM_007381 & 1.8 \\
\hline Hadh & Hydroxyacyl-coenzyme A dehydrogenase & NM_008212 & 2.0 \\
\hline$H a d h b$ & Hydroxyacyl-coenzyme A dehydrogenase, beta subunit & NM_145558 & 1.7 \\
\hline Acsl1 & Acyl-CoA synthetase long-chain family member 1 & NM_007981 & 1.8 \\
\hline Acsm3 & Acyl-CoA synthetase medium-chain family member 3 , transcript variant 2 & NM_21441 & 1.9 \\
\hline \multicolumn{4}{|c|}{ Mitochondrial activity } \\
\hline $\operatorname{Cox} 5 b$ & Cytochrome $c$ oxidase, subunit $\mathrm{Vb}$ & NM_009942 & 2.2 \\
\hline $\operatorname{Cox} 7 a 1$ & Cytochrome $c$ oxidase, subunit VIIa 1 & NM_009944 & 2.6 \\
\hline $\operatorname{Cox} 8 b$ & Cytochrome $c$ oxidase, subunit VIIIb & NM_007751 & 7.2 \\
\hline \multicolumn{4}{|l|}{ Fatty acid synthesis } \\
\hline Fasn & Fatty acid synthase & NM_007988 & 3.2 \\
\hline Acaca/Accl & Acetyl-coenzyme A carboxylase alpha & NM_13336 & 1.9 \\
\hline \multicolumn{4}{|l|}{ Thermogenesis } \\
\hline Ucpl & Uncoupling protein 1 & NM_009463 & 628.8 \\
\hline Cidea & Cell death-inducing DNA fragmentation factor, alpha subunit-like effector A & NM_007702 & 18.1 \\
\hline Cptlb & Carnitine palmitoyltransferase $\mathrm{Ib}$ & NM_009948 & 4.7 \\
\hline Ucp 2 & Uncoupling protein 2 & NM_011671 & 1.7 \\
\hline Dio2 & Deiodinase, iodothyronine, type II & NM_010050 & 3.7 \\
\hline \multicolumn{4}{|c|}{ Prostaglandin synthesis } \\
\hline $\operatorname{Ptgs} 2 / \operatorname{Cox}-2$ & Prostaglandin-endoperoxide synthase 2 /cyclooxygenase- 2 & NM_011198 & 4.4 \\
\hline Ptges & Prostaglandin E synthase & NM_022415 & 1.6 \\
\hline Ptges 2 & Prostaglandin E synthase 2 & NM_133783 & 1.7 \\
\hline \multicolumn{4}{|l|}{ BAT differentiation } \\
\hline Cebpb & CCAAT/enhancer binding protein, beta & NM_009883 & 2.0 \\
\hline
\end{tabular}

WAT (epididymal fat pad) was extracted from wild-type and $\mathrm{Mstn}^{-/-}$mice fed a chow diet

Genes were separated on basis of functions as indicated and were upregulated by at least 1.5 -fold

Acadl and Acsl6 was maintained in WAT, BAT and skeletal muscle of $\mathrm{Mstn}^{-1-}$ mice fed an HFD, no significant increase occurred in WAT (Fig. 3a-c), BAT (Fig. 3d-f) or skeletal muscle (ESM Fig. 1a-c) tissue from HFD-fed wild-type mice. Consistent with the increase of fatty acid oxidation enzyme abundance (Fig. 3), we also observed a large increase in fatty acid oxidation in WAT, liver (Fig. $3 \mathrm{~g}$ ) and primary myotubes cultures from $\mathrm{Mstn}^{-1-}$ mice (ESM Fig. 1f). In addition, and consistent with enhanced fatty acid oxidation, we also found a significant reduction of NEFA (Fig. 3h) in $\mathrm{Mstn}^{-/-}$mice compared with wild-type controls.
Increased expression of genes involved in mitochondrial activity in peripheral tissues of $\mathrm{Mstn}^{-/}$mice Microarray analysis revealed increased expression of genes encoding mitochondrial cytochrome $\mathrm{c}$ oxidase enzyme subunits $\mathrm{Vb}$ (Cox5b), VIIa1 (Cox7a1) and VIIIb (Cox8b) (Table 1). Quantitative PCR analysis also identified an approximately sixfold and tenfold induction of Cptla and Cpt2 expression respectively, in WAT from $\mathrm{Mstn}^{-1-}$ mice fed regular chow diet, compared with wild-type controls on a chow diet (Fig. 2f, g). Importantly, HFD feeding resulted in further upregulation of Cptla and Cpt2 in WAT from Mstn ${ }^{-1-}$ mice. However, no such increase was seen in WAT from wild-type 


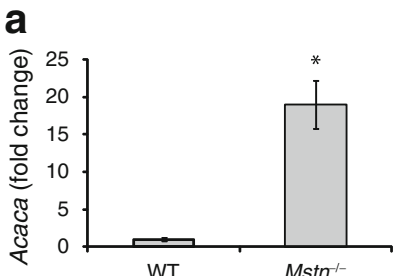

b

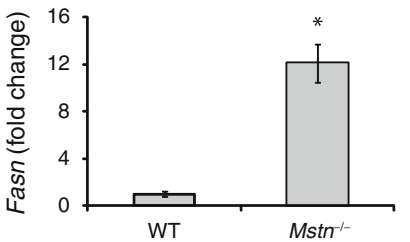

C

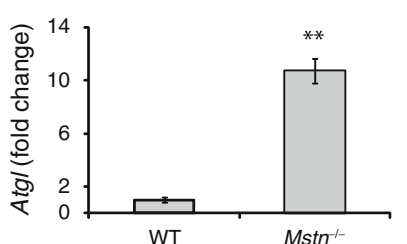

e

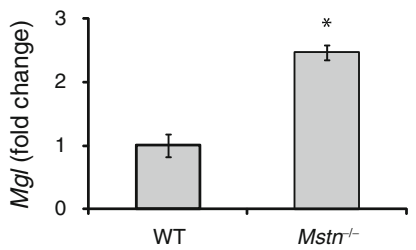

f
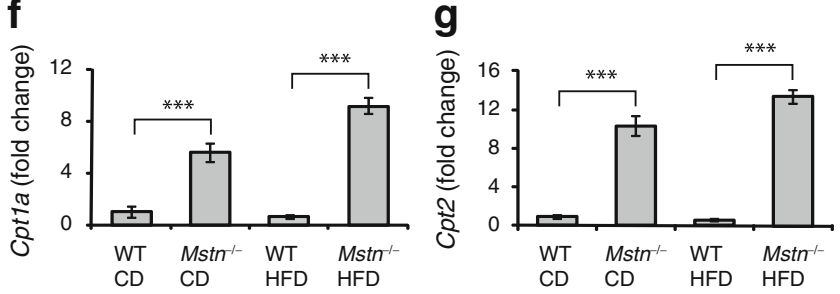

Fig. 2 Increased expression of genes involved in lipolysis, lipid synthesis and mitochondrial activity in $\mathrm{Mstn}^{-/-}$mice. a Relative mRNA expression levels of Acaca, (b) Fasn, (c) Atgl (also known as Pnpla2), (d) $H s l$ (also known as Lipe), (e) $M g l$ (also known as $M g l l$ ), (f) Cpt1a and (g) Cpt2 normalised to Gapdh mRNA expression in WAT (epididymal) as measured by qPCR in wild-type and $\mathrm{Msth}^{-/-}$ mice after 12 weeks of chow diet (CD) and HFD treatment. Values are mean \pm SEM (error bars); $n=6 ; * p<0.05, * * p<0.01, * * * p<0.001$

mice fed an HFD. A modest, yet significant, increase in Cpt1a and Cpt2 (ESM Fig. 1d, e) expression was also observed in skeletal muscle tissue from chow diet- and HFD-fed $\mathrm{Mstn}^{-/-}$mice compared with the respective wildtype controls. Taken together, these data suggest that mitochondrial activity is increased in $\mathrm{Mstn}^{-1-}$ WAT. We propose that this may be one mechanism through which lack of myostatin improves lipid metabolism.

Upregulation of crucial transcriptional factors and BATspecific proteins in $\mathrm{Mstn}^{--}$WAT BAT is primarily responsible for burning lipids, which, via uncoupling proteins such as UCP1, results in dissipation of excess energy as heat [21]. Consistent with this, we found significantly elevated (by $\sim 1{ }^{\circ} \mathrm{C}$ ) body temperature in $\mathrm{Mstn}^{-/-}$mice compared with wild-type mice (Fig. 4a). Since we observed elevated body temperature, increased expression of genes that encode for fatty acid oxidative enzymes and increased $\beta$-oxidation of fatty acids in $\mathrm{Mstn}^{-1-}$ mice, we next determined whether there was increased accumulation of BAT-like cells in $\mathrm{Mstn}^{-1-}$ WAT. Subsequent immunocytochemistry revealed a BAT-like phenotype, with a predominance of small cells with rich cytoplasmic staining, multilocular lipid droplet appearance (Fig. 4b) and pronounced UCP1 production in $\mathrm{Mstn}^{-/-}$WAT (Fig. 4c). Furthermore, qPCR analysis revealed increased expression of $U c p 1, U c p 2$ and $U c p 3$ (Fig. 4d), and of genes involved in BAT specification and thermogenesis, including Ppara, Pparb, Pparg and Pgc-1 $\alpha$, in $\mathrm{Mstn}^{-/-}$WAT; this increase a

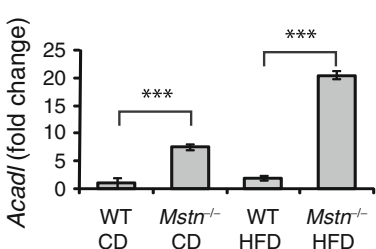

C

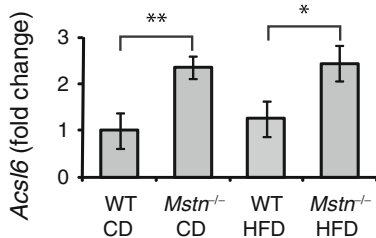

e

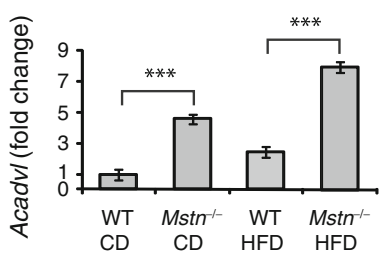

g

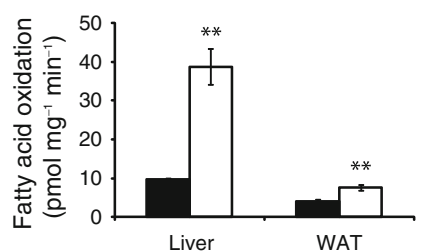

b

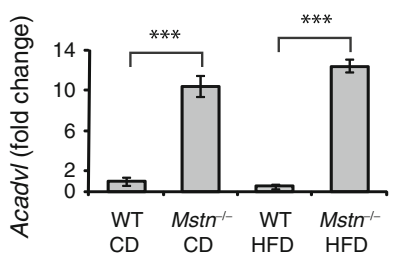

d

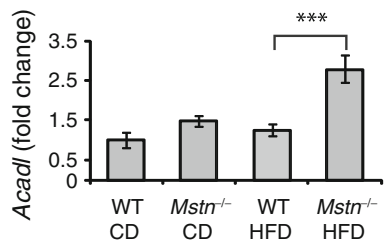

f

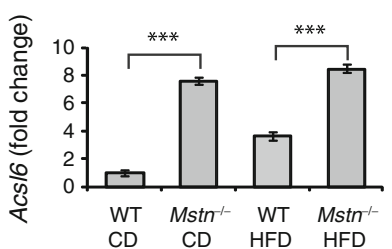

h

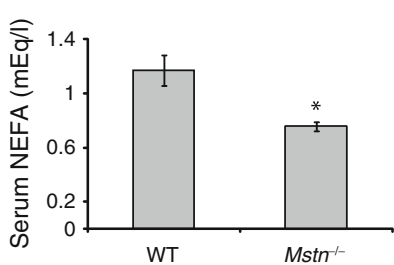

Fig. 3 Lack of myostatin leads to increased fatty acid oxidation in WAT and liver. a Relative mRNA expression levels of Acadl, (b) Acadvl and (c) Acsl6 in WAT (epididymal fat-pad), and of (d) Acadl, (e) Acadvl and (f) Acsl6 in BAT, normalised to Gapdh mRNA expression as measured by qPCR in wild-type (WT) and $\mathrm{Mstn}^{-/-}$mice after 12 weeks of chow diet (CD) and HFD treatment. g Fatty acid oxidation in liver and WAT (epididymal) in wild-type (black bars) and $\mathrm{Mstn}^{-/-}$mice (white bars), normalised to protein content. h Serum NEFA in wild-type and $\mathrm{Mstn}^{-1-}$ mice. Values are mean \pm SEM (error bars); $n=6 ; * p<0.05,{ }^{*} p<<0.01, * * * p<0.001$ 
a

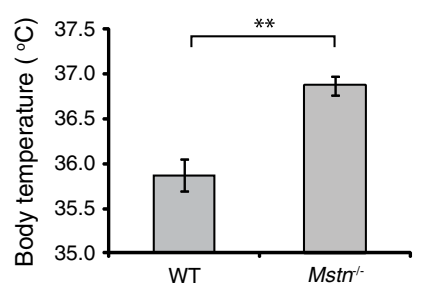

b

b

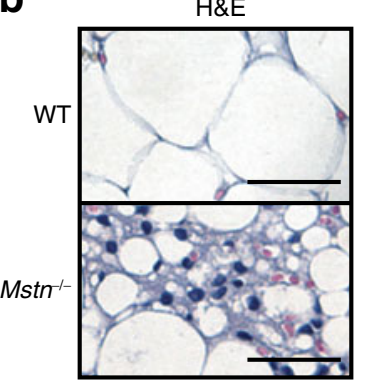

C

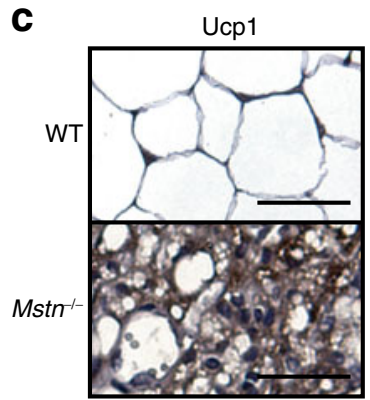

\section{d}
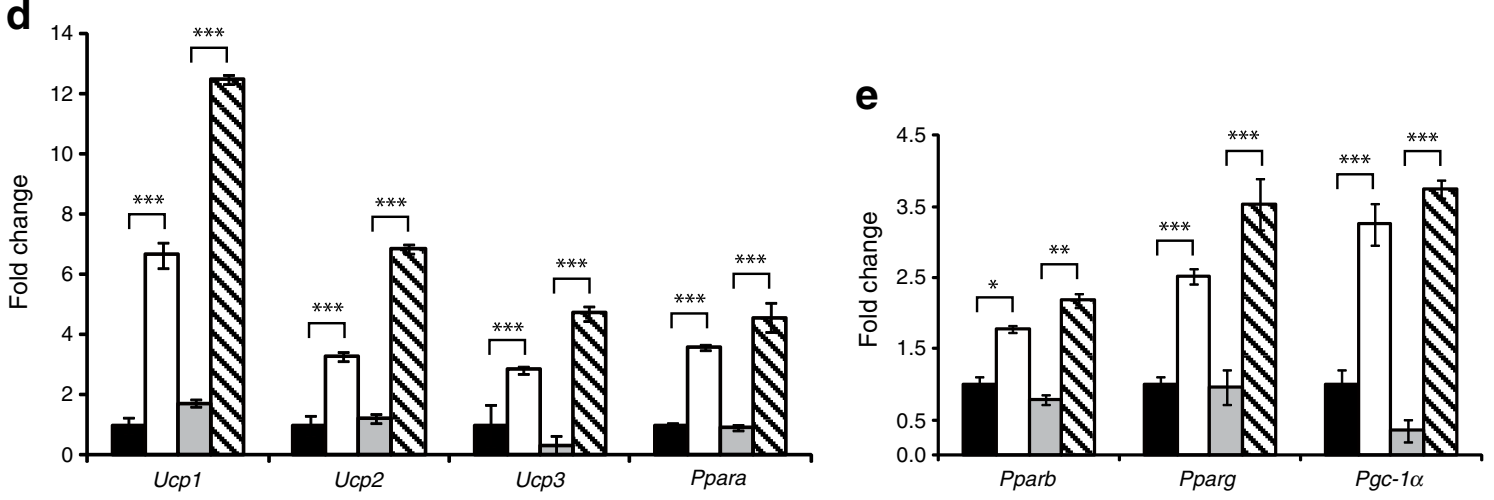

Fig. 4 Enhanced thermogenesis and energy expenditure in WAT from $\mathrm{Msth}^{-/-}$mice. a Rectal body temperature of wild-type (WT) and $\mathrm{Mstn}^{-/-}$mice. b Haematoxylin and eosin staining of WAT from wildtype and $\mathrm{Mstn}^{-/-}$mice. c Immunohistochemistry analysis of UCP1 localisation in WAT from wild-type and $\mathrm{Mstn}^{-/-}$mice. b, c Scale bars $50 \mu \mathrm{m}$, magnification $\times 20$. d, e qPCR analysis of the relative mRNA expression of genes as indicated, normalised to Gapdh mRNA expression in WAT (epididymal) of wild-type and $\mathrm{Mstn}^{-/-}$mice after 12 weeks of chow diet and HFD treatment. Black bars, wild-type chow diet; grey bars, wild-type HFD; white bars, $\mathrm{Mstn}^{-/-}$chow diet; hatched bars, $\mathrm{Mstn}^{-/-}$HFD. Values are mean \pm SEM (error bars); $n=6$; $* p<0.05, * * p<0.01, * * * p<0.001$ was further enhanced upon HFD feeding (Fig. 4d, e). Importantly, we did not observe the multilocular phenotype (Fig. 4b) or increased UCP1 abundance (Fig. 4c) in WAT from wild-type mice. Moreover, no increase in $U c p 1, U_{c p}$, Ucp3, Ppara, Pparb, Pparg and Pgc-1 $\alpha$ expression was detected upon HFD feeding in wild-type mice; in fact, expression of the above genes remained relatively low in wild-type mice WAT, irrespective of diet regimen (Fig. 4d, e). Elevated levels of cyclooxygenase-2 (COX-2), a ratelimiting enzyme in prostaglandin synthesis, contributes to the development of a BAT-like or 'beige' phenotype in WAT [13]. Therefore we next analysed COX-2 levels via immunocytochemistry and qPCR in WAT tissue from wildtype and $\mathrm{Mstn}^{-/-}$mice. The results revealed strong induction of COX-2 abundance and expression of $\operatorname{Ptgs} 2$ (Cox2) in $\mathrm{Mstn}^{-1-}$ mice WAT, but not in WAT from wild-type mice (Fig. 5a, b). Consistent with elevated Ptgs2 and Ptges expression (Fig. 5b, e), we also observed enhanced release of prostaglandin, Prostaglandin E2 $\left(\mathrm{PGE}_{2}\right)$ and 6-ketoprostaglandin $\mathrm{F} 1 \alpha\left(\mathrm{PGF}_{1 \alpha}\right)$ from $\mathrm{Mstn}^{-/-}$mice WAT explants compared with wild-type controls (Fig. 5c, d).

Treatment with myostatin antagonist alleviates diet-induced obesity in mice To determine whether or not postnatal inactivation of myostatin has any therapeutic benefit, wildtype mice were fed a chow diet or HFD for 12 weeks in the absence (saline) or presence of sActRIIB, which has been previously shown to block myostatin signalling. Despite similar energy intake (Fig. 6a), wild-type mice fed an HFD gained more body weight than wild-type mice on a chow diet (Fig. 6b). Moreover, wild-type mice fed an HFD in conjunction with sActRIIB had reduced body weight gain compared with that of wild-type mice fed an HFD only. In fact the body weight gain observed in wild-type mice fed an HFD and treated with sActRIIB was comparable with control chow diet-fed wild-type mice (Fig. 6b). As expected, wild-type mice on an HFD had significantly increased WAT tissue weight (Fig. 6c) and enhanced adipocyte hypertrophy (Fig. 6d, e). However, sActRIIB treatment significantly reduced WAT weight gain (Fig. 6c) and the increased adipocyte size observed following HFD feeding alone (Fig. 6d, e). As expected, chronic HFD feeding resulted in increased serum triacylglycerol content (from 1.88 to $2.53 \mathrm{mmol} / \mathrm{l}$ ). In contrast, a significant reduction of serum triacylglycerol was observed in sActRIIB-treated wild-type mice fed an HFD (from 2.53 to $1.91 \mathrm{mmol} / 1, p<0.05)$. Interestingly, there was no significant difference in serum triacylglycerol levels between 


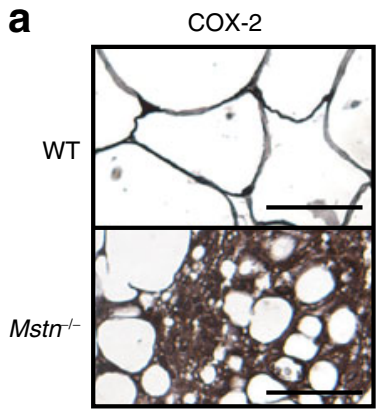

b
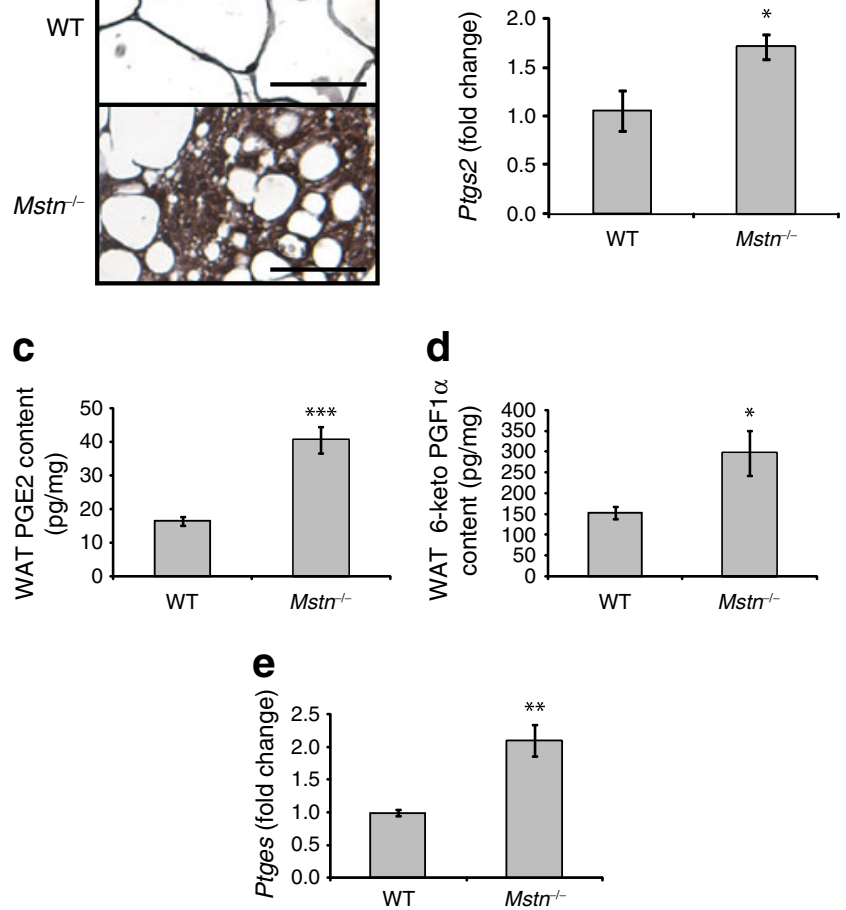

Fig. 5 Enhanced COX-2-mediated prostaglandin signalling in WAT from $\mathrm{Msth}^{-/-}$mice. a Immunohistochemistry analysis of COX-2 localisation in WAT from wild-type (WT) and $\mathrm{Mstn}^{-/-}$mice. Scale bars $50 \mu \mathrm{m}$, magnification $\times 20$. b qPCR analysis of the relative mRNA expression of Ptgs 2 in WAT of $\mathrm{Mstn}^{-/-}$and wild-type mice. $\mathbf{c}$ The levels of PGE2 and (d) and PGF1 $\alpha$ released from $\mathrm{Mstn}^{-1-}$ and wild-type mice WAT explants. e qPCR analysis of the relative mRNA expression of Ptges. mRNA expression (b, e) was normalised to Gapdh mRNA expression in WAT from wild-type and $\mathrm{Mstn}^{-/-}$mice. Values are mean \pm SEM (error bars); $n=6 ;{ }^{*} p<0.05,{ }^{* *} p<0.01,{ }^{* * *} p<0.001$

wild-type mice fed a chow diet and HFD-fed mice treated with sActRIIB (1.88 vs $1.91 \mathrm{mmol} / \mathrm{l})$.

Subsequent qPCR analysis revealed significantly reduced Ucp 1, Ucp 2, Pgc-1 $\alpha$, Ppara, Pparg and Ptgs2 expression in WAT from HFD-fed wild-type mice compared with control wild-type mice on a chow diet (Fig. 6f-h). However, in WAT from HFD-fed wild-type mice treated with sActRIIB, we observed significantly elevated expression of Ucp1, Ucp2,

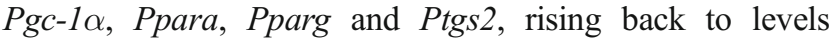
comparable with those observed in control wild-type mice on a chow diet (Fig. $6 \mathrm{f}-\mathrm{h}$ ). Therefore postnatal inactivation of myostatin results in a similar WAT gene expression profile to that observed in $\mathrm{Mstn}^{-/-}$mice fed HFD.

\section{Discussion}

$\mathrm{Msth}^{-1-}$ mice had atrophied adipocytes and failed to accumulate fat in WAT even when fed an HFD (Fig. 1).
Since this does not appear to be due to redistribution of fat in peripheral tissues of $\mathrm{Mstn}^{-/-}$mice [14], we reasoned that it could be due to increased energy expenditure. Consistent with this hypothesis, we found significantly increased expression of genes critical to mitochondrial activity/ function and fatty acid oxidation in the peripheral tissues of $\mathrm{Msth}^{-1-}$ mice (Fig. 3, ESM Fig. 1). In addition, two recent publications have also confirmed an increase in total energy expenditure in myostatin-deficient mice [22, 23], suggesting that increased fatty acid oxidation and total energy expenditure could be key reasons for reduced adiposity in $\mathrm{Mstn}^{-/-}$mice. However, in a recent study, Guo et al. [17] showed that deletion of myostatin specifically in adipose tissue had no significant effects on adipose tissue fat accumulation or metabolic homeostasis. Importantly, myostatin expression is low in adipose tissue [1] compared with skeletal muscle and liver [1, 24], thus conditional deletion of adipose tissue-specific expression of myostatin may not have a dramatic impact on total circulatory levels of myostatin. Moreover, as myostatin functions in an endocrine fashion, we speculate that myostatin synthesised and secreted by muscle and liver (in adipose tissue-specific knockout mice) was sufficient to maintain the biological function of myostatin, possibly explaining why the above authors failed to observe increased muscle mass and reduced fat accumulation in their mice [17]. Here, we used a strain of knockout mice in which expression of functional myostatin is absent. We observed increased mitochondrial activity and fatty acid oxidation consistently in WAT, skeletal muscle and liver. We do not believe that the increased fatty acid oxidation in WAT is a secondary effect of the increased muscle mass observed in $\mathrm{Msth}^{-/-}$mice, as we detected specific upregulation of genes related to increased mitochondrial activity, both in WAT and skeletal muscle. We also hypothesise that increased AMP-activated protein kinase (AMPK) activity [14] and enhanced PPAR pathway signalling (Fig. 4) in $\mathrm{Mstn}^{-/-}$mice are the molecular reason for the increased fatty acid oxidation. Certainly, AMPK activation has been shown to increase fatty acid oxidation $[25,26]$. Similarly, overexpression of PPAR $\beta$ or PPAR $\beta$ agonist treatment resulted in increased mitochondrial number and activity, as well as enhanced abundance of enzymes involved in fatty acid oxidation, and resulting fatty acid oxidation [27]. Consistent with this finding, our unpublished results indicate that although $\mathrm{Mstn}^{-/-}$muscles have increased MHC type IIB-expressing fast-twitch fibres [28], they are metabolically oxidative in nature, since these fibres have increased mitochondrial number and activity (S. Lokireddy, C. McFarlane, M. Sharma and R. Kambadur, unpublished data). Furthermore, histochemical results show that $\mathrm{Mstn}^{-/}$ MHC IIB muscle fibres possess increased succinate dehydrogenase activity, which is consistent with increased 

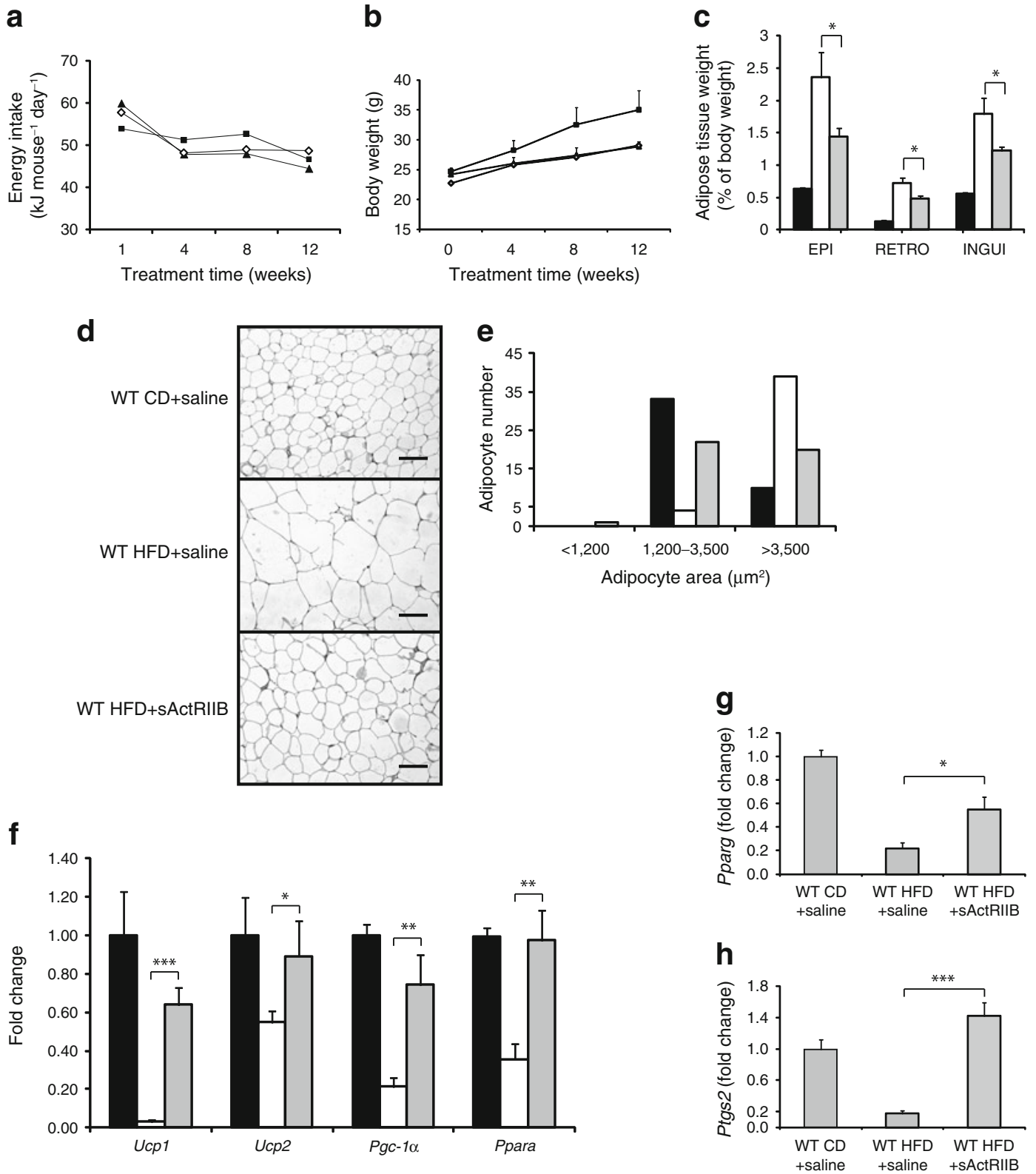

Fig. 6 Postnatal inactivation of myostatin results in an improved metabolic phenotype. a Energy intake (in kJ) and (b) body weight increase (black triangles, wild-type chow diet+saline; black squares, wild-type HFD+saline; white diamonds, wild-type HFD+sActRIIB), as well as (c) epididymal (EPI), retroperitoneal (RETRO) and inguinal (INGUI) fat-pad weight observed in wild-type (WT) mice fed a chow diet or HFD in the presence of saline or sActRIIB. c Black bars, wildtype chow diet+saline; white bars, wild-type HFD+saline; grey bars, wild-type HFD + sActRIIB. d Haematoxylin and eosin staining (scale

oxidative activity in fast-twitch fibres (S. Lokireddy, C. McFarlane, M. Sharma and R. Kambadur, unpublished data). In addition to WAT and skeletal muscle, we also noted increased fatty acid oxidation in liver, corroborating the recent publication by Wilkes et al., who showed decreased liver steatosis in myostatin-deficient mice following HFD feeding [29]. Interestingly, overabundance of activated bars $100 \mu \mathrm{m}$, magnification $\times 10$ ) and (e) quantification of individual adipocyte area in WAT isolated from wild-type mice fed a chow diet (CD) or HFD in the presence of saline or sActRIIB; key to bars as above (c). $\mathbf{f}$ qPCR analysis of the relative mRNA expression of genes as indicated (key to bars as above [c]), and of (g) Pparg and (h) Ptgs2, normalised to Gapdh mRNA expression in WAT from wild-type mice fed a chow diet or HFD in the presence of saline or sActRIIB. Values are mean \pm SEM (error bars); $n=6 ;{ }^{*} p<0.05,{ }^{* *} p<0.01,{ }^{*} * * p<0.001$

Akt in mice leads to increased fast glycolytic fibres in skeletal muscle and increased fatty acid oxidation [30], much like what we observed in $\mathrm{Mstn}^{-1-}$ mice. Interestingly, these mice have a $30 \%$ reduction of myostatin levels. Hence it is possible that increased fast glycolytic fibres in $\mathrm{Msth}^{-/-}$mice could result in increased liver fatty acid oxidation. 
Ucp 1 was significantly upregulated in $\mathrm{Mstn}^{--}$WAT (Table 1 and Fig. 4). In addition, the expression of members of the PPAR family (Ppara, Pparb and Pparg) and of Pgc$1 \alpha$, as well as that of crucial factors for controlling adipocyte differentiation, lipolysis, fatty acid oxidation and thermogenesis, was also upregulated in WAT of $\mathrm{Mstn}^{-1}$ mice (Table 1 and Figs 2 and 3). Multilocular adipocyte morphology was also observed in WAT of $\mathrm{Mstn}^{-1-}$ mice (Fig. 4). Together these observations strongly suggest that WAT of $\mathrm{Mstn}^{-1-}$ mice has acquired characteristics of BAT. However, further work will be necessary to determine whether or not the appearance of BAT in $\mathrm{Mstn}^{-1-}$ mice WAT is accompanied by increased neural innervation and vascularisation, which would be consistent with the increased metabolic activity observed. Although published reports have described the transient appearance of BAT in WAT in humans and in experimental animal models [31, 32], the growth factor signalling mechanisms that regulate the conversion/formation of brown adipocytes is not known. Our results suggest that myostatin could be one such novel growth factor capable of determining BAT content in WAT. Our search for the molecular mechanism(s) behind myostatin-induced regulation of BAT formation in WAT found that absence of myostatin induces COX-2 production. Recently, it was shown that COX-2 is a downstream effector of $\beta$-adrenergic signalling in WAT and that upregulation of COX-2 is required and sufficient for de novo production of BAT in WAT deposits. Once induced, COX-2 promoted thermogenic gene expression, including Ucp1, Cidea, Cpt1b and Dio2, and enhanced prostaglandin release from WAT, resulting in conversion of WAT mesenchymal progenitors into a BAT-like or 'beige' phenotype [13]. We also observed elevated thermogenic gene expression (Table 1 and Fig. 4) and enhanced prostaglandin release from $\mathrm{Mstn}^{-/-}$mice WAT explants (Fig. 5), suggesting that similar mechanisms to that seen following $\beta$-adrenergic stimulation may be responsible for the development of BAT in Mstn ${ }^{-1-}$ WAT. Consistent with the enhanced BAT phenotype, we also observed increased expression of Ucp 1, Ucp2 and Ucp3 in $\mathrm{Mstn}^{-/-}$WAT, genes that are responsible for uncoupling and the dissipation, as heat, of the energy generated in mitochondria, resulting in increased body temperature (Fig. 4a). It is important to mention that although we observed an enhanced BAT-like phenotype in $\mathrm{Mstn}^{-1-}$ mice WAT, we did not find any significant changes in BAT morphology or weights (data not shown).

Further results presented here demonstrate that treatment with a myostatin antagonist (sActRIIB) in vivo replicates the anti-obesity phenotype observed in $\mathrm{Mstn}^{-1-}$ mice, suggesting that blockade of myostatin may be an effective anti-obesity therapy. However, it remains unclear at this stage whether the effects of sActRIIB on WAT gene expression are primary or secondary to the increased muscle mass observed following sActRIIB treatment [33]. It is noteworthy that, in an independent animal trial, treatment with myostatin-specific antibodies had no effect on fat mass accretion in adipose tissue [22]. This result suggests that not all myostatin antagonists will increase muscle mass and reduce body fat content. Here we used sActRIIB because it has been successfully shown to increase muscle growth in $M d x$ (also known as $D m d$ ) mice [34], to increase insulin sensitivity during diet-induced obesity [35] and to reduce cachectic muscle wasting [36], much like what is seen in $\mathrm{Mstn}^{-1-}$ mice. However, sActRIIB can bind to several additional ligands in circulation [37, 38], so it may function by inactivating ligands other than myostatin. Therefore, before sActRIIB can be used as an effective anti-obesity therapeutic, its target specificity will need to be determined.

The current results demonstrate that inactivation of myostatin leads to increased resistance to HFD-induced obesity in mice. We propose that increased quantities of 'brown-like adipose tissue' in WAT and increased peripheral tissue fatty acid oxidation through enhanced PPAR signalling are the two main mechanisms through which mice are protected against HFD-induced obesity in response to lack of or inhibition of myostatin. These findings highlight myostatin antagonists as a novel class of potential antiobesity drugs, which function by increasing energy expenditure, rather than limiting food/fat intake.

Acknowledgements We would like to thank the Agency for Science, Technology and Research (A*STAR) for funding this project. Thanks also to J. Swain (Singapore Institute for Clinical Sciences, Singapore) for her comments on the manuscript and R. Choo (Singapore Institute for Clinical Sciences, Singapore) for his invaluable help with statistical analysis of the data presented in this manuscript. Further thanks to K. Wong (School of Biological Sciences, Nanyang Technological University, Singapore) for help with performing biochemical analysis.

Contribution statement $\mathrm{CZ}, \mathrm{CM}, \mathrm{SL}, \mathrm{SM}, \mathrm{XG}, \mathrm{PDG}, \mathrm{MS}$ and RK planned and conceived the idea, performed research work, wrote the manuscript and approved the final version of the manuscript for publication.

Duality of interest The authors declare that there is no duality of interest associated with this manuscript.

\section{References}

1. McPherron AC, Lawler AM, Lee SJ (1997) Regulation of skeletal muscle mass in mice by a new TGF-beta superfamily member. Nature 387:83-90

2. Sharma M, Kambadur R, Matthews KG et al (1999) Myostatin, a transforming growth factor-beta superfamily member, is expressed in heart muscle and is upregulated in cardiomyocytes after infarct. J Cell Physiol 180:1-9 
3. McPherron AC, Lee SJ (1997) Double muscling in cattle due to mutations in the myostatin gene. Proc Natl Acad Sci USA 94:12457-12461

4. Kambadur R, Sharma M, Smith TP, Bass JJ (1997) Mutations in myostatin (GDF8) in double-muscled Belgian Blue and Piedmontese cattle. Genome Res 7:910-916

5. Clop A, Marcq F, Takeda H et al (2006) A mutation creating a potential illegitimate microRNA target site in the myostatin gene affects muscularity in sheep. Nat Genet 38:813-818

6. Schuelke M, Wagner KR, Stolz LE et al (2004) Myostatin mutation associated with gross muscle hypertrophy in a child. $\mathrm{N}$ Engl J Med 350:2682-2688

7. McPherron AC, Lee SJ (2002) Suppression of body fat accumulation in myostatin-deficient mice. J Clin Invest 109:595-601

8. Zhao B, Wall RJ, Yang J (2005) Transgenic expression of myostatin propeptide prevents diet-induced obesity and insulin resistance. Biochem Biophys Res Commun 337:248-255

9. Feldman BJ, Streeper RS, Farese RV Jr, Yamamoto KR (2006) Myostatin modulates adipogenesis to generate adipocytes with favorable metabolic effects. Proc Natl Acad Sci USA 103:1567515680

10. Cigolini M, Cinti S, Bosello O, Brunetti L, Bjorntorp P (1986) Isolation and ultrastructural features of brown adipocytes in culture. J Anat 145:207-216

11. Guerra C, Koza RA, Walsh K, Kurtz DM, Wood PA, Kozak LP (1998) Abnormal nonshivering thermogenesis in mice with inherited defects of fatty acid oxidation. J Clin Invest 102:17241731

12. Guerra C, Koza RA, Yamashita H, Walsh K, Kozak LP (1998) Emergence of brown adipocytes in white fat in mice is under genetic control. Effects on body weight and adiposity. J Clin Invest 102:412-420

13. Vegiopoulos A, Muller-Decker K, Strzoda D et al (2010) Cyclooxygenase- 2 controls energy homeostasis in mice by de novo recruitment of brown adipocytes. Science 328:1158-1161

14. Zhang C, McFarlane C, Lokireddy S et al (2011) Myostatindeficient mice exhibit reduced insulin resistance through activating the AMP-activated protein kinase signalling pathway. Diabetologia 54:1491-1501

15. Denton RM, Randle PJ (1967) Concentrations of glycerides and phospholipids in rat heart and gastrocnemius muscles. Effects of alloxan-diabetes and perfusion. Biochem J 104:416-422

16. Frayn KN, Maycock PF (1980) Skeletal muscle triacylglycerol in the rat: methods for sampling and measurement, and studies of biological variability. J Lipid Res 21:139-144

17. Guo T, Jou W, Chanturiya T, Portas J, Gavrilova O, McPherron AC (2009) Myostatin inhibition in muscle, but not adipose tissue, decreases fat mass and improves insulin sensitivity. PLoS One 4: e4937

18. Hirschey MD, Shimazu T, Goetzman E et al (2010) SIRT3 regulates mitochondrial fatty-acid oxidation by reversible enzyme deacetylation. Nature 464:121-125

19. Wensaas AJ, Rustan AC, Lovstedt K et al (2007) Cell-based multiwell assays for the detection of substrate accumulation and oxidation. J Lipid Res 48:961-967

20. Dilger AC, Spurlock ME, Grant AL, Gerrard DE (2010) Myostatin null mice respond differently to dietary-induced and genetic obesity. Anim Sci J 81:586-593
21. Golozoubova V, Hohtola E, Matthias A, Jacobsson A, Cannon B, Nedergaard J (2001) Only UCP1 can mediate adaptive nonshivering thermogenesis in the cold. FASEB J 15:2048-2050

22. Bernardo BL, Wachtmann TS, Cosgrove PG et al (2010) Postnatal PPARdelta activation and myostatin inhibition exert distinct yet complimentary effects on the metabolic profile of obese insulinresistant mice. PLoS One 5:e11307

23. Choi SJ, Yablonka-Reuveni Z, Kaiyala KJ, Ogimoto K, Schwartz MW, Wisse BE (2011) Increased energy expenditure and leptin sensitivity account for low fat mass in myostatin-deficient mice. Am J Physiol Endocrinol Metab 300:E1031-E1037

24. Li ZB, Kollias HD, Wagner KR (2008) Myostatin directly regulates skeletal muscle fibrosis. J Biol Chem 283:19371-19378

25. Ruderman NB, Dean D (1998) Malonyl CoA, long chain fatty acyl CoA and insulin resistance in skeletal muscle. J Basic Clin Physiol Pharmacol 9:295-308

26. Canto C, Gerhart-Hines Z, Feige JN et al (2009) AMPK regulates energy expenditure by modulating $\mathrm{NAD}^{+}$metabolism and SIRT1 activity. Nature 458:1056-1060

27. Wang YX, Lee CH, Tiep S et al (2003) Peroxisome-proliferatoractivated receptor delta activates fat metabolism to prevent obesity. Cell 113:159-170

28. Hennebry A, Berry C, Siriett V et al (2009) Myostatin regulates fiber-type composition of skeletal muscle by regulating MEF2 and MyoD gene expression. Am J Physiol Cell Physiol 296:C525C534

29. Wilkes JJ, Lloyd DJ, Gekakis N (2009) Loss-of-function mutation in myostatin reduces tumor necrosis factor alpha production and protects liver against obesity-induced insulin resistance. Diabetes 58:1133-1143

30. Izumiya Y, Hopkins T, Morris C et al (2008) Fast/glycolytic muscle fiber growth reduces fat mass and improves metabolic parameters in obese mice. Cell Metab 7:159-172

31. Cousin B, Cinti S, Morroni M et al (1992) Occurrence of brown adipocytes in rat white adipose tissue: molecular and morphological characterization. J Cell Sci 103:931-942

32. Cypess AM, Lehman S, Williams G et al (2009) Identification and importance of brown adipose tissue in adult humans. N Engl $\mathrm{J}$ Med 360:1509-1517

33. Lee SJ, Reed LA, Davies MV et al (2005) Regulation of muscle growth by multiple ligands signaling through activin type II receptors. Proc Natl Acad Sci U S A 102:18117-18122

34. Morine KJ, Bish LT, Selsby JT et al (2010) Activin IIB receptor blockade attenuates dystrophic pathology in a mouse model of Duchenne muscular dystrophy. Muscle Nerve 42:722-730

35. Akpan I, Goncalves MD, Dhir R et al (2009) The effects of a soluble activin type IIB receptor on obesity and insulin sensitivity. Int J Obes (Lond) 33:1265-1273

36. Zhou X, Wang JL, Lu J et al (2010) Reversal of cancer cachexia and muscle wasting by ActRIIB antagonism leads to prolonged survival. Cell 142:531-543

37. Sako D, Grinberg AV, Liu J et al (2010) Characterization of the ligand binding functionality of the extracellular domain of activin receptor type IIb. J Biol Chem 285:21037-21048

38. Souza TA, Chen X, Guo Y et al (2008) Proteomic identification and functional validation of activins and bone morphogenetic protein 11 as candidate novel muscle mass regulators. Mol Endocrinol 22:2689-2702 